\title{
TUPLE OF OPERATORS AND EPSILON SUPERCYCLICITY
}

\author{
Bahmann Yousefi ${ }^{1}$, Javad Izadi ${ }^{2}$ \\ ${ }^{1,2}$ Department of Mathematics \\ Payame Noor University \\ P.O. Box 19395-3697, Tehran, IRAN
}

Abstract: In this paper we prove that if a tuple of operators is $\epsilon$-supercyclic for all $\epsilon>0$, then it is supercyclic.

AMS Subject Classification: 47B37, 47B33

Key Words: supercyclic vector, $\epsilon$-supercyclicity

\section{Introduction}

From now on, let $T_{1}, T_{2}, \ldots, T_{n}$ be commutative bounded linear operators on a Banach space $X$.

Definition 1.1. Let $\mathcal{T}=\left(T_{1}, T_{2}, \ldots, T_{n}\right)$ be an n-tuple of operators acting on an infinite dimensional Banach space $X$. We will let

$$
\mathcal{F}_{\mathbf{T}}=\left\{T_{1}{ }^{k_{1}} T_{2}{ }^{k_{2}} \ldots T_{n}{ }^{k_{n}}: k_{i} \geq 0, i=1, \ldots, n\right\}
$$

be the semigroup generated by $\mathcal{T}$. For $x \in X$, the orbit of $x$ under the tuple $\mathcal{T}$ is the set

$$
\operatorname{Orb}(\mathcal{T}, x)=\left\{S x: S \in \mathcal{F}_{\mathbf{T}}\right\} .
$$

A vector $x$ is called a hypercyclic vector for $\mathcal{T}$ if $\operatorname{Orb}(\mathcal{T}, x)$ is dense in $X$ and in this case the tuple $\mathcal{T}$ is called hypercyclic. Also, a vector $x$ is called a supercyclic vector for $\mathcal{T}$ if $\operatorname{COrb}(\mathcal{T}, x)$ is dense in $X$ and in this case the tuple $\mathcal{T}$ is called supercyclic.

Received: June 14, 2014

(C) 2014 Academic Publications, Ltd.

$\S_{\text {Correspondence author }}$ url: www.acadpubl.eu 
Definition 1.2. Let $\epsilon \in(0,1)$ and $x \in X$. If for every non zero vector $y \in X$, there exist integers $k_{1}, \ldots, k_{n}$ and $\lambda \in \mathbb{C}$ such that

$$
\left\|\lambda T_{1}^{k_{1}} T_{2}{ }^{k_{2}} \ldots T_{n}{ }^{k_{n}} x-y\right\|<\epsilon\|y\|,
$$

then the vector $x$ is called $\epsilon$-supercyclic for the n-tuple $\mathcal{T}=\left(T_{1}, T_{2}, \ldots, T_{n}\right)$. A tuple of operators is $\epsilon$-supercyclic if it admits an $\epsilon$-supercyclic vector.

For some sources on these topics see [1-20].

\section{Main Results}

In this section we prove that if a tuple is $\epsilon$-supercyclic for all $\epsilon>0$, then it is supercyclic. We will use the idea of Theorem 2.1 in [20] for a tuple of operators. We will denote $\mathbb{N} \cup\{0\}$ by $\mathbb{N}_{0}$. We will use $S C(\mathcal{T})$ for the collection of supercyclic vectors for a tuple of operators $\mathcal{T}$.

Theorem 2.1. Let $X$ be a separable infinite dimensional Banach space and $\mathcal{T}=\left(T_{1}, T_{2}, \ldots, T_{n}\right)$ be a tuple of operators $T_{1}, T_{2}, \ldots, T_{n}$. Then $\mathcal{T}$ is supercyclic, if and only if for any two non-void open sets $U$ and $V$, there exist $m_{i} \geq 1$ for $i=1, \ldots, n$ and and $\lambda \in \mathbb{C} \backslash\{0\}$ such that $\lambda T_{1}^{m_{1}} \ldots T_{n}^{m_{n}}(U) \cap V \neq \varnothing$.

Proof. Suppose that $x$ is a supercyclic vector for $\mathcal{T}$, hence $\operatorname{COrb}(\mathcal{T}, x)$ is dense in $X$ and so there exist $\lambda_{1} \in \mathbf{C} \backslash\{0\}$ and $m_{i} \in \mathbb{N} ; i=1, \ldots, n$ such that $\lambda_{1} T_{1}^{m_{1}} \ldots T_{n}^{m_{n}} x \in U$. Let $y=\lambda_{1} T_{1}^{m_{1}} \ldots T_{n}^{m_{n}} x$. Since $\operatorname{COrb}(\mathcal{T}, x)$ is also dense in $X$, thus $\lambda T_{1}^{m_{1}} \ldots T_{n}^{m_{n}} y \in V$ for some $\lambda \in \mathbb{C} \backslash\{0\}$ and $m_{i} \in \mathbb{N} ; i=1, \ldots, n$, hence $\lambda T_{1}^{m_{1}} \ldots T_{n}^{m_{n}}(U) \cap V \neq \varnothing$. Conversely, fix an enumeration $\left\{B_{n}: n \in \mathbb{N}\right\}$ of the open balls in $X$ with rational radii, and centers in a countable dense subset of $X$. By the continuity of the operators $T_{1}, T_{2}, \ldots, T_{n}$, the sets

$$
G_{k}=\bigcup\left\{\lambda T_{1}^{-m_{1}} \ldots T_{n}^{-m_{n}}\left(B_{k}\right): m_{i} \geq 0 ; i=1, \ldots, n, \lambda \in \mathbf{C}\right\}
$$

are open. Since for any two non-void open sets $U$ and $V$, there exist $m_{i} \geq 1$; $i=1, \ldots, n$, and $\lambda \in \mathbf{C} \backslash\{0\}$ such that $\lambda T_{1}^{m_{1}} \ldots T_{n}^{m_{n}}(U) \cap V \neq \varnothing$, thus one can see that $S C(\mathcal{T})$ is exactly equal to $\bigcap\left\{G_{k}: k \in \mathbb{N}\right\}$ that is dense in $X$. This completes the proof.

Theorem 2.2. Let $X$ be a separable infinite dimensional Banach space and $\mathcal{T}=\left(T_{1}, T_{2}, \ldots, T_{n}\right)$ be the n-tuple of operators $T_{1}, T_{2}, \ldots, T_{n}$. If for every $\epsilon>0, \mathcal{T}$ is $\epsilon$-supercyclic, then $\mathcal{T}$ is supercyclic. 
Proof. Suppose that $U$ and $V$ are nonempty open subsets of $X$. Let $u \in U$ and $v \in V$ be two nonzero vectors, and consider

$$
0<\delta<\min \{\|u\|,\|v\|\}
$$

small enough such that $B(u, \delta) \subset U$ and $B(v, \delta) \subset V$. Choose

$$
\epsilon<\delta /(6 \max \{\|u\|,\|v\|\})
$$

and let $x \in X$ be an $\epsilon$-supercyclic vector for $\mathcal{T}$. This implies that there exist nonnegative integers $k_{1}^{(0)}, \ldots, k_{n}^{(0)}$ and $\lambda \in \mathbb{C}$ such that

$$
\left\|\lambda T_{1}^{k_{1}^{(0)}} T_{2}^{k_{2}^{(0)}} \ldots T_{n}^{k_{n}^{(0)}} x-u\right\|<\epsilon\|u\|<\delta .
$$

Hence

$$
\lambda T_{1}^{k_{1}^{(0)}} T_{2}^{k_{2}^{(0)}} \ldots T_{n}^{k_{n}^{(0)}} x \in B(u, \delta) \subset U .
$$

We want to show that

$$
V \cap\left\{\lambda T_{1}^{k_{1}} T_{2}{ }^{k_{2}} \ldots T_{n}{ }^{k_{n}} x: k_{i} \geq 0, i=1, \ldots, n\right\}
$$

contain infinitely many elements. Suppose on the contrary that it contains only the elements $\lambda T_{1}^{k_{1}^{(i)}} T_{2}^{k_{2}^{(i)}} \ldots T_{n}^{k_{n}^{(i)}} x$ for $i=1, \ldots, k$. As we saw earlier, for each $v^{\prime} \in B\left(v, \frac{2 \delta}{3}\right)$ there exist integers $k_{1}\left(v^{\prime}\right), \ldots, k_{n}\left(v^{\prime}\right)$ which satisfies

$$
\left\|\lambda T_{1}^{k_{1}\left(v^{\prime}\right)} T_{2}^{k_{2}\left(v^{\prime}\right)} \ldots T_{n}^{k_{n}\left(v^{\prime}\right)} x-v^{\prime}\right\| \leq \epsilon|\lambda|\left\|v^{\prime}\right\| \leq 2 \epsilon|\lambda|\|v\|<\frac{\delta}{3} .
$$

Hence

$$
\lambda T_{1}^{k_{1}\left(v^{\prime}\right)} T_{2}^{k_{2}\left(v^{\prime}\right)} \ldots T_{n}^{k_{n}\left(v^{\prime}\right)} \in\left\{\lambda T_{1}^{k_{1}^{(i)}} T_{2}^{k_{2}^{(i)}} \ldots T_{n}^{k_{n}^{(i)}}: i=1, \ldots, k\right\},
$$

because

$$
\left\|\lambda T_{1}^{k_{1}\left(v^{\prime}\right)} \ldots T_{n}^{k_{n}\left(v^{\prime}\right)} x-v\right\| \leq\left\|\lambda T_{1}^{k_{1}\left(v^{\prime}\right)} \ldots T_{n}^{k_{n}\left(v^{\prime}\right)} x-v^{\prime}\right\|+\left\|v^{\prime}-v\right\|<\delta .
$$

Therefore

$$
B\left(v, \frac{2 \delta}{3}\right) \subset \bigcup_{i=1}^{k} B\left(\lambda T_{1}^{k_{1}^{(i)}} T_{2}^{k_{2}^{(i)}} \ldots T_{n}^{k_{n}^{(i)}} x, \frac{\delta}{3}\right),
$$

that is a contradiction since $X$ is infinite dimensional. Thus, indeed the set

$$
V \cap\left\{\lambda T_{1}^{k_{1}} T_{2}{ }^{k_{2}} \ldots T_{n}{ }^{k_{n}} x: k_{i} \geq 0, i=1, \ldots, n\right\}
$$


contain infinitely many elements and so the set

$$
B(v, \delta) \cap\left\{\lambda T_{1}^{k_{1}} \ldots T_{n}^{k_{n}} x: k_{i} \geq 0, i=1, \ldots, n\right\}
$$

has infinite elements. In particular, there exist $k_{i} \in \mathbb{N}_{0}$ satisfying $k_{i} \geq k_{i}{ }^{(0)}$ for $i=1, \ldots, n$ such that $\lambda T_{1}^{k_{1}} T_{2}{ }^{k_{2}} \ldots T_{n}{ }^{k_{n}} x \in V$. Thus we get

$$
\lambda T_{1}^{k_{1}-k_{1}{ }^{(0)}} \ldots T_{n}^{k_{n}-k_{n}{ }^{(0)}} T_{1}^{k_{1}{ }^{(0)}} \ldots T_{n}^{k_{n}{ }^{(0)}} x=\lambda T_{1}^{k_{1}} T_{2}{ }^{k_{2}} \ldots T_{n}{ }^{k_{n}} x
$$

which belongs to

$$
\lambda T_{1}^{k_{1}-k_{1}{ }^{(0)}} T_{2}^{k_{2}-k_{2}{ }^{(0)}} \ldots T_{n}^{k_{n}-k_{n}{ }^{(0)}}(U) \cap V .
$$

Now by Theorem 2.1 the proof is complete.

\section{References}

[1] C. Badea, S. Grivaux and V. Muller, Epsilon-hypercyclic operators, Institute of Mathematics, AS CR, Prague, (2008), 7-24.

[2] J. Bes, and A. Peris, Hereditarily hypercyclic operators, J. Func. Anal., 167, No. 1 (1999), 94-112.

[3] P. S. Bourdon and J. H. Shapiro, Hypercyclic operators that commute with the Bergman backward shift, Trans. Amer. Math. Soc., 352, No. 11 (2000), 5293-5316.

[4] R. M. Gethner and J. H. Shapiro, Universal vectors for operators on spaces of holomorphic functions, Proc. Amer. Math. Soc., 100 (1987), 281-288.

[5] G. Godefroy, J. H. Shapiro, Operators with dense, invariant cyclic vector manifolds, Journal of Functional Analysis, 98 (1991), 229-269.

[6] K. Goswin, G. Erdmann, Universal families and hypercyclic operators, Bulletin of the American Mathematical Society, 35 (1999), 345-381.

[7] H. N. Salas, Hypercyclic weighted shifts, Trans. Amer. Math. Soc., 347 (1995), 993-1004.

[8] S. Shkarin, Non-sequential weak supercyclicity and hypercyclicity, Journal of Functional Analysis, 242, No. 1 (2007), 37-77. 
[9] B. Yousefi, and H. Rezaei, Hypercyclicity on the algebra of Hilbert-Schmidt operators, Results in Mathematics, 46 (2004), 174-180.

[10] B. Yousefi and H. Rezaei, Some necessary and sufficient conditions for Hypercyclicity Criterion, Proc. Indian Acad. Sci. (Math. Sci.), 115, No. 2 (2005), 209-216.

[11] B. Yousefi and A. Farrokhinia, On the hereditarily hypercyclic vectors, Journal of the Korean Mathematical Society, 43, No. 6 (2006), 1219-1229.

[12] B. Yousefi, H. Rezaei and J. Doroodgar, Supercyclicity in the operator algebra using Hilbert-Schmidt operators, Rendiconti Del Circolo Matematico di Palermo, Serie II, Tomo LVI (2007), 33-42.

[13] B. Yousefi and H. Rezaei, Hypercyclic property of weighted composition operators, Proc. Amer. Math. Soc., 135, No. 10 (2007), 3263-3271.

[14] B. Yousefi and S. Haghkhah, Hypercyclicity of special operators on Hilbert function spaces, Czechoslovak Mathematical Journal, 57, No. 132 (2007), 1035-1041.

[15] B. Yousefi and H. Rezaei, On the supercyclicity and hypercyclicity of the operator algebra, Acta Mathematica Sinica, 24, No. 7 (2008), 1221-1232.

[16] B. Yousefi and R. Soltani, Hypercyclicity of the adjoint of weighted composition operators, Proc. Indian Acad. Sci. (Math. Sci.), 119, No. 3 (2009), 513-519.

[17] B. Yousefi, Hereditarily transitive tuples, Rend. Circ. Mat. Palermo, 2011, DOI 10.1007/s12215-011-0066-y (2011).

[18] B. Yousefi and J. Izadi, Weighted composition operators and supercyclicity criterion, International Journal of Mathematics and Mathematical Sciences, 2011, DOI 10.1155/2011/514370, (2011).

[19] B. Yousefi and J. Izadi, Para-chaotic tuples of operators, The Australian Journal of Mathematical Analysis and Applications, 9, No. 1 (2012), 245249.

[20] B. Yousefi and J. Izadi, On the supercyclicity criterion for a tuple of operators, Wulfenia Journal, 20, No. 8 (2013), 227-236. 
